\title{
Optimal structural synthesis of agricultural legged robot with minimal damage on soil
}

\author{
Sayat Ibrayev,"*, Nutpulla Jamalov², Arman Ibrayeva², and Gaukhar \\ Mukhambetkaliyeva ${ }^{2}$ \\ ${ }^{1}$ Institute of Mechanics and Mechanical Engineering named after academician U.A. Joldasbekov, \\ Almaty, Kazakhstan \\ ${ }^{2}$ Al-Farabi Kazakh National University, Almaty, Kazakhstan
}

\begin{abstract}
Optimal structural synthesis of agricultural legged robot is carried out, that causes minimal damage on soil and provide the most favorable conditions for plant growth. A rational structure of a legged robot with orthogonal propel based on kinematic decoupling of the motion is proposed. Most of traditionally used walking robots have universal "insect type" structure with multiple actuators to be synchronized which result in complex control. The alternative design is realizing a concept of functional independence of the actuators when each actuator is responsible for specified purpose: the main actuators are responsible for rectilinear translational motion of cabine/chassis whereas another group of actuators are responsible for adaptation purposes and anothers participate in turning/maneuvering. This allows to carry out the cabine/hull shifting and turning with a minimum number of actuators and simplified control. A new kinematic equivalent scheme of turning mechanism was proposed in order to optimize turning modes of the robot. The proposed planar model allows to determine the optimal parameters of the robot by applying multicriteria synthesis methods of parallel robot manipulators based on isotropy, maneuverability and other criteria.
\end{abstract}

\section{Introduction}

Legged machines that can adapt to off-road conditions and irregular terrain, have a number of advantages over traditional wheeled and tracked vehicles. The benefits of using legged robot (LR) in agriculture are discussed in [1]. In particular, they cause the least damage to the soil, in comparison with wheeled and tracked vehicles, due to the discrete track on the ground. Meanwhile, attaining to increase productivity of agricultural machines leads to an increase of machine weight that cause increased soil pressure and unfavorable conditions for plant growth. Various designing concepts of LR have been proposed by scientific teams in developed countries by now [2 - 5]. However many of them are extremely ineffective in terms of power consumption (energy efficiency) and complexity of the control system. In particular, more than ten actuators and multi-level control were used for turning of such systems [6]. The alternative design is

* Corresponding author: sayat_m.ibrayev@mail.ru 
- to consider LR as consisting of functional blocks as

- the locomotor system,

- the adaptation mechanism,

- and the mechanism of turning/maneuvering

- and decouple the actuators so that each actuator has a specified functional destination/purpose [7 - 13].

In this paper, a rational structure of LR is proposed which allows to carry out the robot cabine/hull shifting and rotation with a minimum number of actuators. At the same time adjusting the chassis height and foot adaptation on the rough terrain is carried out by another group of actuators. A kinematically equivalent planar scheme of the robot is proposed in order to simplify the study of the turning modes. As the result the optimal geometric parameters of the robot can be determined by applying properly developed multicriteria synthesis methods of closed-loop robot manipulators based on isotropy, maneuverability and other criteria.

\section{Motion decoupling and separation of the actuator functions concept applied to LR}

Turning gait of a six-legged walking robot using tripod gait is considered in [14] studying two types of general gaits: rotation and spinning. Various approaches for turning mode study had been applied also in $[15,16]$ considering traditional LR of insect like structure. But the lapse in the structure of the most LR is underconstrained design and multiple static ambiguity (indetermination). The number of active drives are much more than the number of degrees of freedom and on the other hand the locomotion is often possible using much smaller number of actuators. For example in Hexapod-type robots (with three actuators per leg) during tripod gait the number of d.o.f is equal six, whereas the number of active drives is 9. (Of course this is taking into account just supporting legs; but if all legs are on the ground then the number of actuators is equal 18, whereas the number of d.o.f. yet again 6). This results in parasitic loads in drives (the latter act "against each other"), increasing of joint reactions, foot slippage etc.

The alternative design is to apply the concept of motion decoupling and principle of actuator functions separation $[1,7,9,17-19]$ which results in energy efficiency and simplifying control. The example of implementation of this principle is LR with rectilinear guiding leg mechanisms (see layout of LR shown in Figure 1). Another leg mechanism is shown in Figure 2 where the output link (supporting link) generates rectilinear and translational motion. Planar six-bar linkage $A B C D T Q R$ is used as the leg mechanism with a coupler link $T Q$ (output link or supporting link) as the output link with foot point $S$. Due to the translational motion of link $T Q$ each point of this link describes the same (identic) trajectory with rectilinear segment ("stepping cycle"). Therefore foot point $S$ generates "stepping cycle" and the trajectory remains exactly the same while adjusting the height $h$. In order to adjust the straight line height $h$ the adaptation link ES is mounted on the output link $T Q$. So this example demonstrates actuators functions separation: the input link (crank) $A B$ is responsible for rectilinear and translational motion of the robot body/chassis and the additional input link $E S$ is necessary for adaptation to the ground irregularity.

When the crank angle $\varphi_{A D}=(O \vec{Y}, \overrightarrow{A B})$ of six-bar mechanism is changed within the limits $\varphi_{0} \leq \varphi_{A B} \leq \varphi_{0}+\Phi_{s p}$ points $T$ and $Q$ are laid on rectilinear segment of the "stepping cycle" and foot-point $S$ occurs on the support phase.

Further rotation of the crank $A B$ and corresponding change of the crank angle $\varphi_{A B}$ within the limits $\varphi_{0}+\Phi_{S F} \leq \varphi_{A D} \leq \varphi_{0}+\Phi_{S F}+\Phi_{T F}, \Phi_{S F}+\Phi_{T F}=2 \pi$, corresponds to 
transference phase of the "stepping cycle" when foot-point $S$ carries out "carry-over" phase. If $\Phi_{S P}>\pi$, then $\frac{\phi_{Y F}}{\hat{\omega}_{T F}}>1$ and "overlapping" of two legs is provided.

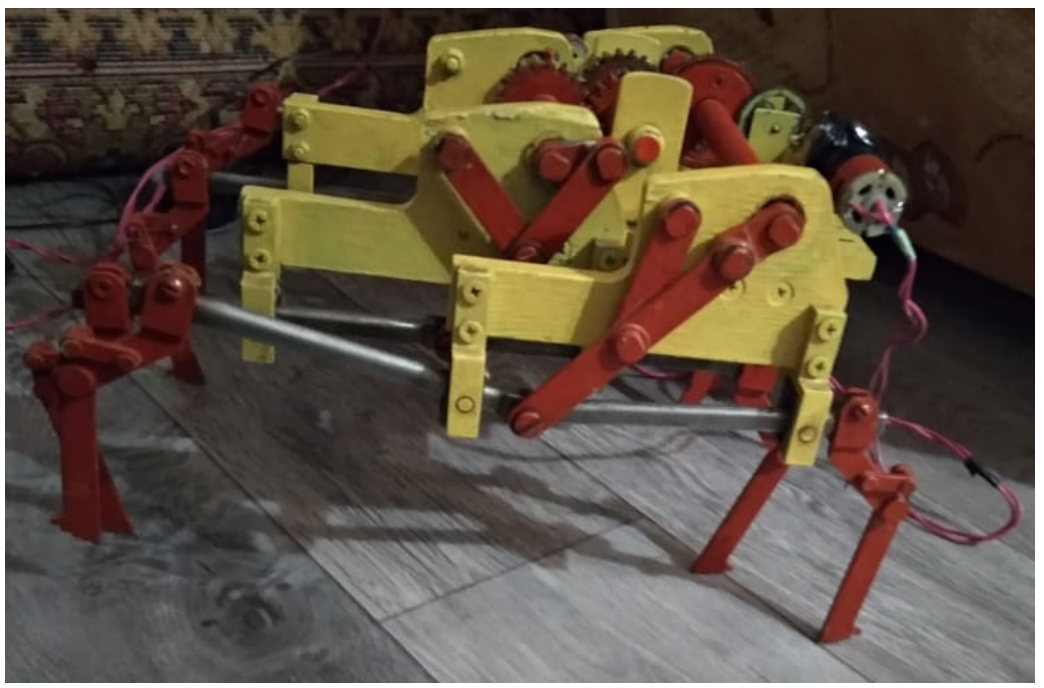

Fig. 1. Layout of LR using rectilinear guiding leg mechanisms.

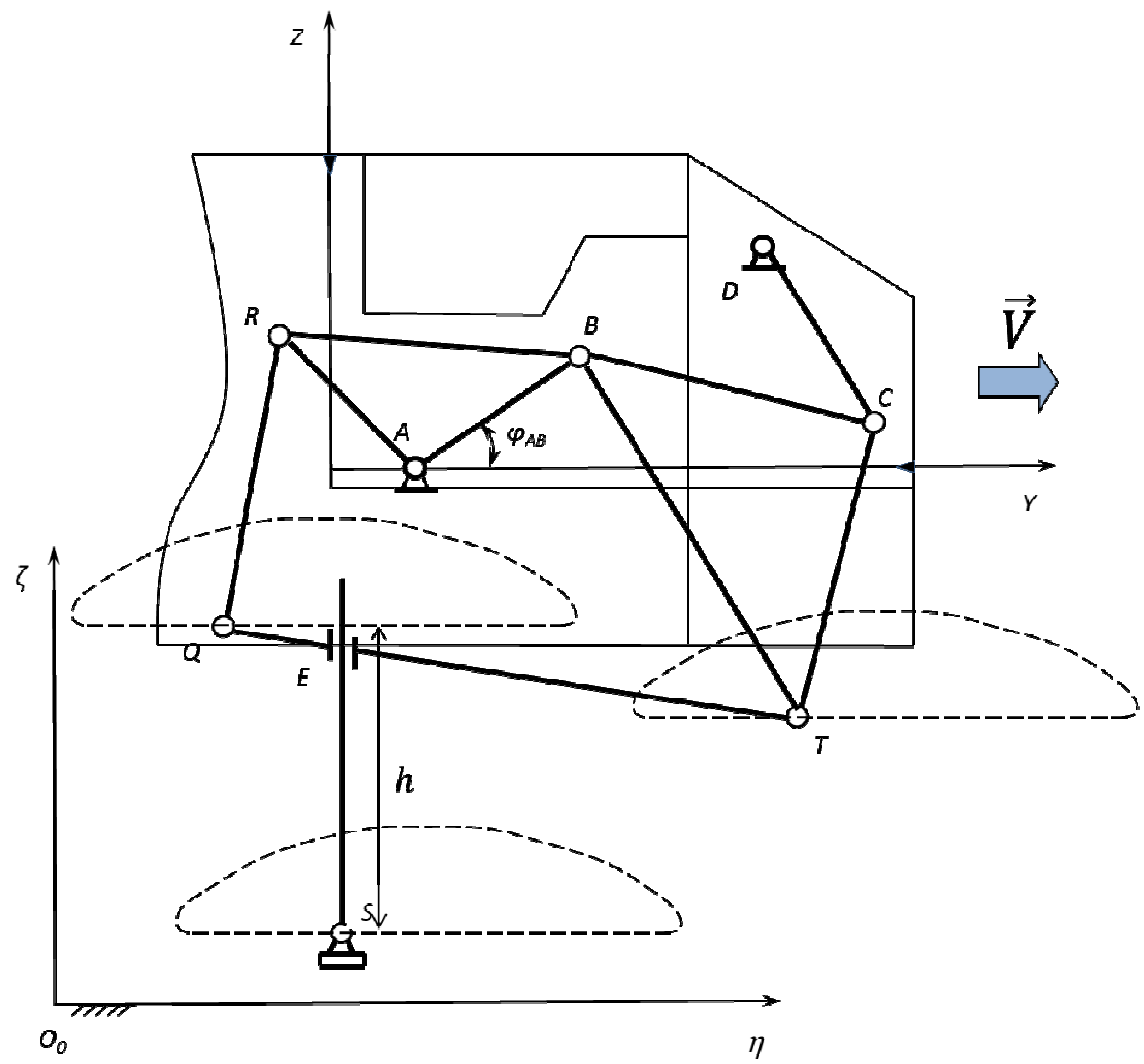

Fig. 2. Leg mechanism of LR with rectilinear and translational motion of support link. 
Note that if the robot body/hull moves horizontally with a velocity $V$, then the coupler $T Q$ and foot-point $S$ move relative to the body/hull with a speed $V_{S}=-V$. So the coupler $T Q$ remains stationary relative to the "ground" coordinate system fixed on supporting surface. As the additional input link ES providing lifting and lowering of the foot in vertical direction does not influence the horizontal motion of the foot $S$, its horizontal velocity relative to the ground will remain always equal to zero.

In order to turn the LR let introduce for each $i$-th leg additional joints $\sigma_{i}$ rotating around the vertical axes $O_{i} z_{i}^{g}$ (Figure 3).
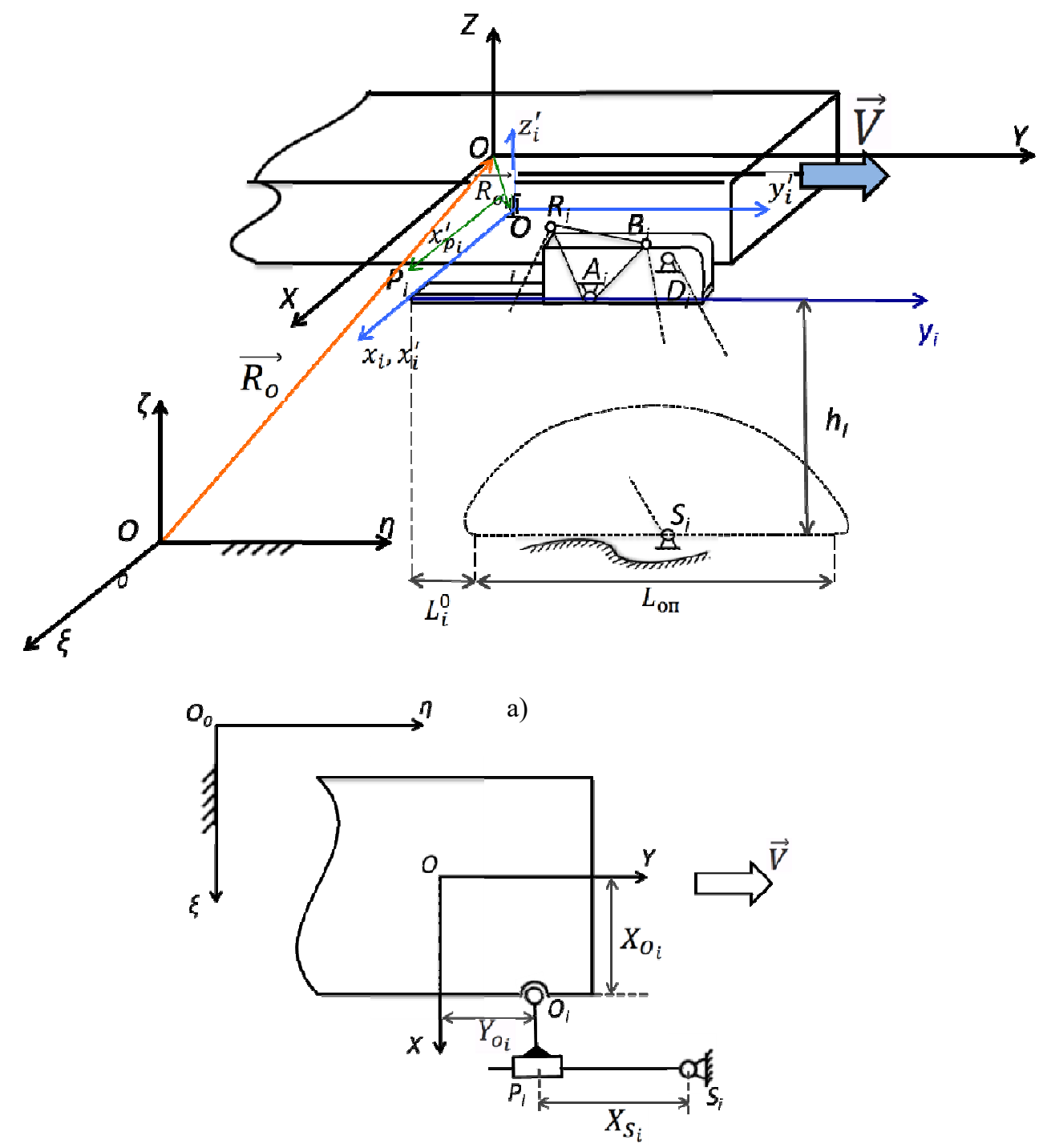

b)

Fig. 3. Leg mechanism number $i$ (a) and the image of the leg motion as the planar RPR kinematic chain (b).

Let introduce the following coordinate systems: 
- $O_{0} \xi \eta \xi$ is an absolute (fixed) coordinate system fixed on the ground (on support surface); the LR body/hull moves along the axis $O_{0} \eta$ with the velocity $V$.

- $O X Y Z$ is a coordinate system, fixed on the body/hull of the LR.

- $O_{i} x_{i}^{l} y^{f} z_{i}^{l}$ is a local coordinate system rigidly fixed on the $i$-th leg, its origin $O_{i}$ coincides with the center of joint $O_{i}$.

- $P_{i} x_{i} y_{i} z_{i}$ is also a local coordinate system fixed rigidly on the $i$-th leg mechanism; the plane $P_{i} y z_{i}$ coincides with the plane of the leg mechanism, the axes are parallel to the axes of the coordinate system $O_{i} x_{i}^{l} y^{l} z_{i}^{l}$ and the origin $P_{i}$ is shifted along the axis $x_{i}^{l}$ by the distance $x_{F_{i}}$.

Let the foot $S_{i}$ be on the ground (leg is in support phase), so that $\xi_{S_{i}}=$ const, $\eta_{s_{i}}=$ const, $\zeta_{s_{i}}=$ const. When the crank $A_{i} B_{i}$ rotates uniformly, i.e. with the constant angular velocity $\dot{q}_{\mathrm{t}}=\dot{\varphi}_{\mathrm{t}}=\omega_{i}=$ const, the foot $S_{i}$ moves uniformly and rectilinearly with respect to the coordinate system $O X Y Z$, i.e. parallel to the axis $P_{i} Y$ with the constant velocity $\mathbf{V}_{S_{1}}=-\mathbf{V}$. Neglecting minor deviations one can assume that the coordinate $y$ of the foot $S_{i}$ depends linearly on generalized coordinate $q_{i}$ :

$$
y_{S_{i}}=y_{s_{i}}^{E}=A_{i}+B_{i} q_{i}
$$

and the coordinates $x$ and $z$ remain constant:

$$
\begin{gathered}
x_{S_{i}}=0, x_{S_{i}}^{e}=x_{P_{i}}^{s}=\text { const, } \\
z_{\Upsilon_{S_{i}}}=z_{S_{i}}^{e}=-h_{i}
\end{gathered}
$$

For rectilinear and uniform motion of the robot body/hull, all cranks $A_{i} B_{i}$ of support legs have to rotate with the same angular velocity $\dot{q}_{\mathrm{i}}=\omega_{i}=\omega$. Then differentiating expression (2) we obtain

$$
y_{s_{i}}=v_{s_{i}}^{y}=B_{i} \hat{q}_{i}=B_{i} \omega_{i}
$$

and therefore

$$
B_{i}=B=-\frac{v}{\omega}=\text { const }
$$

where $V$ is a translational velocity of the robot body/hull: $V_{S_{i}}^{y}=-V$.

For convenience of further study of the turning of the LR we consider the coordinates $y_{i}=y_{s_{i}}$ as conditional generalized coordinates instead of $q_{\bar{i}}$ (Figure $3 b$ ).

\section{Optimal Structural Synthesis of Walking Robot}

Let's consider the turning of the six-legged walking robot based on so-called "tripod gait", when three legs are always in support, and three in transference phase, which then alternate. So in Figure 4 legs 1, 3 and 5 are in support phase, and the 2nd, 4th and 6th legs are in transference phase.

The center of mass of the system must always be inside a "support triangle" $S_{1} S_{3} S_{\mathrm{z}}$ for stabile motion.

If all joints are active, i.e. not only the input kinematic pairs $P_{1}, P_{3}$ and $P_{5}$, but also the joints $O_{1}, O_{3}$ and $O_{5}$ are actuated (placing rotary drives on them) then we have additional generalized coordinates $\alpha_{1}, \alpha_{3}, \alpha_{\mathrm{z}}$, besides $y_{1}, y_{2}$ and $y_{\mathrm{z}}$, where $\alpha_{1}, \alpha_{2}, \alpha_{\mathrm{g}}$ are the rotation angles of the links $O_{i} P_{i}$ about the joints $O_{i}$. So the number of active drives (6) will be 
greater than the number of degrees of freedom $(W=3)$ and we have underconstrained system (Note that the 2-nd, 4-th, 6-th legs are in transference phase and they are not taken into account). This is a common fault in LR design and leads to additional loads on drives (motors interfere with each other working against each other), feet slipping relative to the ground and increasing joint reactions. Therefore only translational kinematic pairs $P_{1}, P_{8}$ and $P_{5}$ (which correspond to the generalized coordinates $y_{1}, y_{2}$ and $y_{5}, y_{i}=S_{i} P_{i}$ ) have to be actuated (driven) and now the number of degrees of freedom $W$ is equal to three in accordance with well-known Malyshev's formula:

$$
W=3 n-2 p_{s}=3 \cdot 7-2 \cdot 9=3 .
$$

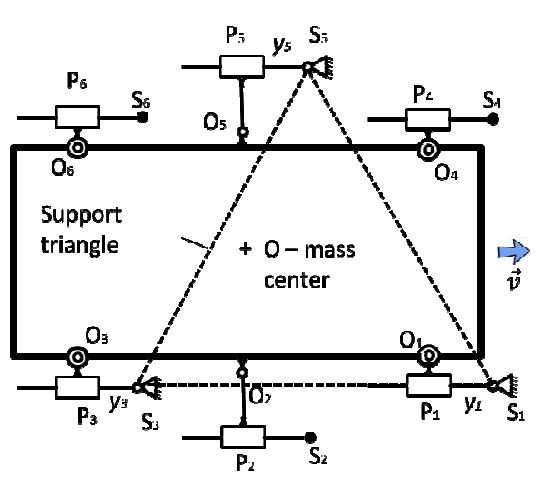

a)

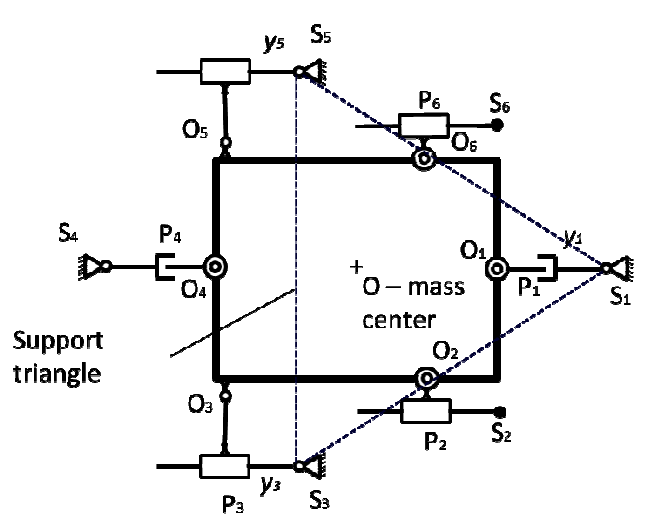

b)

Fig. 4. Kinematic equivalent scheme of six-legged robot based on "tripod-gait".

The turn of the LR in this case will be carried out due to the difference in velocities $y_{1}$, $y_{2}$ and $y_{5}$ (actually in angular velocities $\omega_{1}, \omega_{2}$ and $\omega_{5}$ in the origin scheme). It should be noted that now the conditional generalized coordinate $y_{i}=S_{i} P_{i}$ can take both positive and negative values, since the translational kinematic pairs are just conditional and used just to facilitate the study of turning modes (the 'slider' is only the conditional image of the six-bar leg mechanism). As for the conditional generalized coordinates, as noted above, during the rectilinear-translational motion of the robot body/hull the values of $\dot{y}_{h}$ are negative $\left(y_{t}=-V<0\right)$, here $V(V>0)$ is the body velocity. However while turning the robot, $y_{t}$ may take both negative and positive values. But the $y_{i}$ values are changed within following restrictions

$$
L_{i}^{0} \leq y_{i} \leq L_{i}^{0}+L_{s p}
$$

or

$$
L_{i}^{p}-L_{s p} \leq y_{i} \leq L_{i}^{0}
$$

where $L_{\xi p}$ is the length of the rectilinear segment of the "stepping cycle" trajectory (Figure 3), $L$ is the distance from the axis $P_{i} z_{\mathrm{i}}$ to the straight-line section of the trajectory.

Another important limitation that should be taken into account during the modeling of the LR turning is avoiding singularities. The kinematic chain of the robot must not occur in singular position, when the lines $S_{i} O_{i}$ intersect at one point (Figure 5a), or all $S_{i} O_{i}$ are 
parallel (of course this concerns supporting legs, i.e. only the lines $S_{i} O_{i}$ related to supporting legs) (Figure 5b).
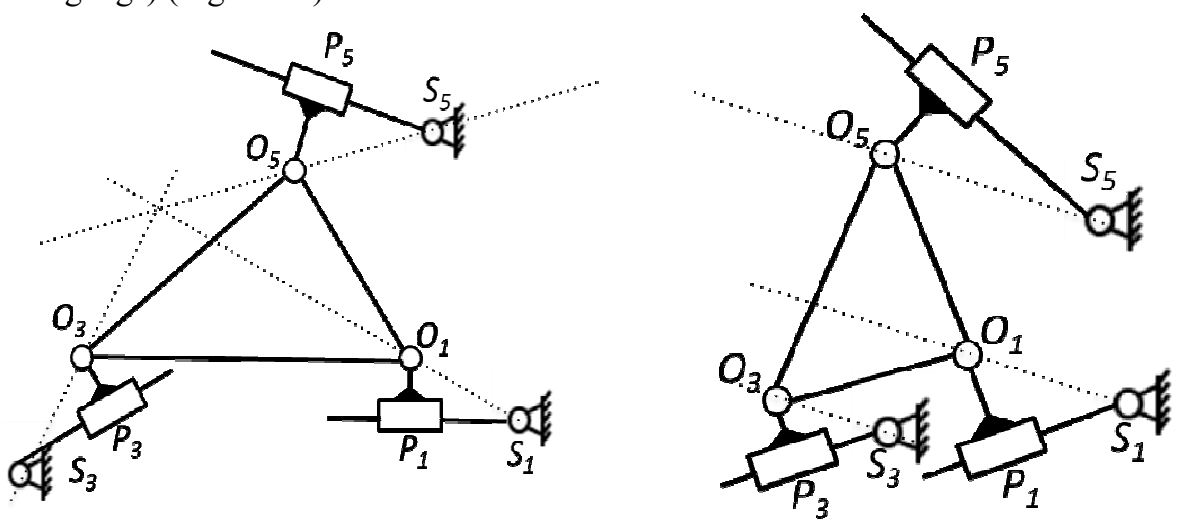

a)

b)

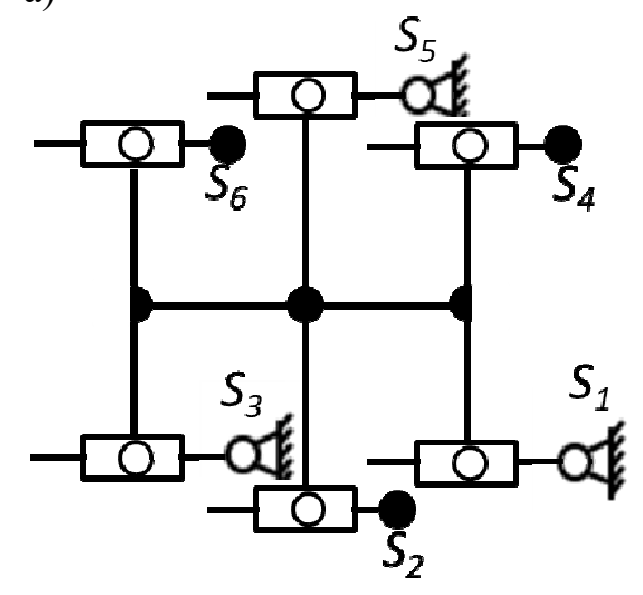

c)

Fig. 5. Configurations of LR in singular positions.

Remark 1. If all $O_{\mathrm{i}} P_{\mathrm{i}}$ are choosen to be equal to zero while designing $\left(O_{i} P_{1}=0\right)$, then LR configuration will be singular at all times of movement of the LR by rectilinear translational motion (Fig. 5c) and the turning from any position will not be possible due to the position ambiguity, i.e. the lack of single-determined configuration.

Remark 2. It should be taken into account during design of LR, that the joints $O_{i}$ (Fig. 3) are introduced not as the input joints (actuated joints), but just to avoid structural redundancy of the robot kinematic chain. The rectilinear-translational motion of the body is possible without these joints, however this will lead to non-correct kinematic structure due to the structural redundancy, since the number of degrees of freedom of the LR kinematic chain will be equal to zero: $W=3 \cdot 4-2 \cdot 6=0$ and the robot turning will not be possible in this case.

Remark 3. When foot-point $S_{j}$ goes into transference phase it does not contact the surface (foot is raised) and then the appropriate joints $O_{j}$ have to be actuated (joints $O_{2}, O_{4}$ and $o_{6}$ in Fig. 2 a with $j=2,4,6$ ). However in practice it is sufficient just to return the link 
$o_{i} P$ (the plane of the leg mechanism, see Figure 1) to the zero ("neutral") position: to the position when the angle $\left(\widehat{O Y,} \widehat{\mathbf{O}_{1} \mathbf{P}_{1}}\right)$, between the link $O_{i} P_{1}$ (leg mechanism plane) and the axis $O Y_{\varepsilon}$ is $90^{\circ}$. Thus it is enough just to place "returning link" (spring for instance) on the joints $\vec{O}_{\mathrm{i}}$ instead of the active drives.

Remark 4. Finally the six-legged walking robot will have only six active drives acting together (instead of 12 in planar scheme and 18 in spatial hexapod-type LR). The robot control aims to ensure the coordinated action of these drives, so reducing the number of drives down to six leads to a significant discharge of LR control. Note that due to the concept of separation of the actuator functions, the LR locomotion control is carried out independently from the adaptation system (the latter includes six additional inputs, but coordination is not required for them, since each leg is adapted individually).

\section{Conclusion}

The optimal structural solution for agricultural LR is proposed, that causes least harm on the ground and the most favorable conditions for plant growth. The concept of decoupled design and separation of the actuators functions leads to the optimal structural design of the LR. This results in the optimal locomotion of the LR from mechanical point of view (optimal power consumption and energy efficiency) using minimal control. The optimal structural design of walking robot with orthogonal propel allowed

- to solve the problem of underconstrained design and multiple static ambiguity (indetermination) of traditional LR;

- and minimize the number of actuators (shorten down to 6 besides 18 in prototype).

Finally the proposed planar model allows to carry out further parametric optimization of the LR on the basis of known methods of multicriteria synthesis of parallel manipulators (based on isotropy, dexterity criteria and others).

\section{References}

1. N. Umnov, Osobennosti mehanizmov shagajushhego tipa dlja ispol'zovanija ih $v$ netradicionnom transporte. Jenciklopedija mashinostroenija v soroka tomah. Chast' 2, vol. 1 (M: Mashinostroenie, 1996)

2. Zh. Baigunchekov, S. Ibrayev, M. Izmambetov, T. Baigunchekov, B. Naurushev, Z. Baigunchekov, et al., Parallel Manipulator of a Class RoboMech. In: Zhang X., Wang N., Huang Y. (eds) Mech. and Mach. Sci. (ASIAN MMS 2016), CCMMS 2016. Lecture Notes in Electrical Engineering, 408, Springer, Singapore (2017)

3. Zh. Baigunchekov, S. Ibrayev, M. Izmambetov, 4th IEEE/IFToMM Int. Conf. ReMAR, Delft University of Technology, The Netherlands (2018)

4. Kim, Sangbae, Wensing, Patrick, Foundations and Trends in Robotics, 5, 117-190 (2017)

5. F. Tedeschi, G. Carbone, Robotics, 3, 181-206 (2014)

6. G. Carbone, M. Ceccarelli, In: Cutting Edge Robotics, V. Kordic, A. Lazinica, M. Merdan, 553-576 (InTech: Vienna, Austria, 2005)

7. Sh.-M. Song, K. Waldron, J. Kenneth, Machines that walk: the adaptive suspension vehicle (Cambridge, Mass, MIT Press, 1989)

8. M. Plecnik, J. Mark McCarthy, Mech. and Mach. Theory, 96. 38 - 51 (2016) 
9. H. Kim, J. Choi, Proc. 14th IFToMM World Congr. TMM, Taipei, Taiwan (2015)

10. G. Chen, B. Jin, Y. Chen, J. Mech. Sci. and Tech., 31 (2017)

11. A. Comănescu, D. Comănescu, I. Dugăeşescu, L. Ungureanu, Proc. 14th IFToMM World Congr., 387-392 (2015)

12. G. Chen, B. Jin, Y. Chen, J. Mech. Sci. And Tech., 31(3), 1401-1411 (2017)

13. G. Haynes, A. Rizzi, Proc. IEEE Int. Conf. Robotics and Automation, 2006, ICRA 2006, 1117-1122 (2006)

14. D. Wettergreen, C. Thorpe, Proc. IEEE/RSJ Int. Conf. Intelligent Robots and Syst., 2,1413 - 1420 (1992)

15. A. Ryan, K. Hunt, Trans. ASME: J. of Mechanisms. Transmissions and Automation in Design, 107, 256 - 261 (1985)

16. S. Song, V. Vohnout, K. Waldron, G. Kinzel, Mechanisms and Mach. Theory, 19, 17 24 (1984)

17. S. Ibraev, N. Imanbaeva, A. Zhauyt, Engineering for rural development, 16. $904-909$ (2017) 